\title{
How well do we care for patients with end stage chronic obstructive pulmonary disease (COPD)? A comparison of palliative care and quality of life in COPD and lung cancer
}

\author{
J M Gore, C J Brophy, M A Greenstone
}

\begin{abstract}
Background-Patients with severe chronic obstructive pulmonary disease (COPD) have a poor quality of life and limited life expectancy. This study examined whether these patients were relatively disadvantaged in terms of medical and social care compared with a group with inoperable lung cancer.

Methods-An open two group comparison was made of 50 patients with severe COPD (forced expiratory volume in one second $\left(F E V_{1}\right)<0.751$ and at least one admission for hypercapnic respiratory failure) and 50 patients with unresectable non-small cell lung cancer (NSCLC). A multi-method design was used involving standardised quality of life tools, semistructured interviews, and review of documentation.
\end{abstract}

Results-The patients with COPD had significantly worse activities of daily living and physical, social, and emotional functioning than the patients with NSCLC $(p<0.05)$. The Hospital Anxiety and Depression Scale (HADS) scores suggested that $90 \%$ of patients with COPD suffered clinically relevant anxiety or depression compared with $52 \%$ of patients with NSCLC. Patients were generally satisfied with the medical care received, but only $4 \%$ in each group were formally assessed or treated for mental health problems. With regard to social support, the main difference between the groups was that, while $30 \%$ of patients with NSCLC received help from specialist palliative care services, none of the patients with COPD had access to a similar system of specialist care. Finally, patients in both groups reported a lack of information from professionals regarding diagnosis, prognosis and social support, although patients' information needs were disparate and often conflicting.

Conclusion-This study suggests that patients with end stage COPD have significantly impaired quality of life and emotional well being which may not be as well met as those of patients with lung cancer, nor do they receive holistic care appropriate to their needs.

(Thorax 2000;55:1000-1006)

Keywords: chronic obstructive pulmonary disease; lung cancer; quality of life; depression; palliative care

Chronic obstructive pulmonary disease (COPD) has been described as a major though neglected medical and social problem in the UK today. ${ }^{1}$ It may affect as many as $18 \%$ of men and $7 \%$ of women between the ages of 40 and 64 years, ${ }^{2}$ although the proportion requiring inpatient treatment is only a fraction of this. Nevertheless, in an average health district COPD will account for 1000 admissions and 25000 primary care consultations annually ${ }^{3}$ and $6.4 \%$ of all male and $3.9 \%$ of all female deaths. ${ }^{4}$ While treatment with bronchodilators and steroids may partially relieve symptoms and oxygen therapy may prolong life, for many patients the course of the disease is one of inexorable decline: a prolonged period of disabling dyspnoea and increasingly frequent hospital admission reflecting deteriorating lung function and usually presaging a premature death. It has long been recognised that, in this group of patients, both quality of life and survival is poor. For instance, in the Nocturnal Oxygen Therapy trial of long term oxygen therapy, disturbances in emotional and social functioning were common and there was a marked impairment in activities of daily living. ${ }^{5}$ In the Medical Research Council trial of domiciliary oxygen, survival of men and women in the control arm was only $42 \%$ and $28 \%$, respectively, at three years. ${ }^{6}$ These poor survival figures are worse than for many common tumours, yet the process and experience of those dying from COPD has received surprisingly little attention.

In marked contrast, when a diagnosis of malignancy is made the focus is not only on maximising survival prospects but how best to support patients through their illness. In patients with a poor prognosis, specialist services often provide a holistic form of care known as palliative care. This has been
13 April 2000

Accepted for publication

17 May 2000 
described as the active total care of patients whose disease is incurable and where the control of physical, psychological, social, and spiritual problems is paramount. ${ }^{7}$ Although many patients might benefit from such an all embracing beneficence, the reality of its practical delivery is almost exclusively confined to malignant disease. ${ }^{8}$ Palliative care is not synonymous with terminal care and it should be apparent that the palliative approachcentring on symptom management, maintenance of a reasonable quality of life, good communication, and practical and emotional support for carers-is necessary for a range of chronic debilitating illnesses and should routinely be an integral part of their management. Although this philosophy has recently been embraced by those dealing with AIDS and degenerative neurological disorders, there is still a huge unmet potential for patients suffering from end stage cardiac ${ }^{9}$ and respiratory diseases. ${ }^{10}$

Respiratory physicians in the UK spend much of their professional lives looking after patients with COPD and lung cancer, usually in the same hospital setting and with the support of the same medical and nursing teams. Nevertheless, our impression was that, among the population of COPD patients who required frequent admission to hospital, the care was unsatisfactory; some patients appeared to have a disproportionately lengthy stay or were readmitted without clear objective signs of deterioration. Others presented in extremis, necessitating rushed decisions about intubation with little information available about their previous quality of life or wishes about resuscitation. Patients with lung cancer, however, seemed to enjoy a more holistic and integrated package of care. We hypothesised that the COPD population might have an equally poor health status, be dissatisfied with their level of care, and have similar palliative needs to the lung cancer population, but that these needs were not recognised or met. We set out to measure their quality of life, psychological morbidity, satisfaction with care (particularly in relation to communication with medical and social professionals), and provision of practical assistance. We aimed to compare the effectiveness in meeting the care needs of both groups. To do this we used a mixture of sociometric and psychological tools, plus in-depth interviews using qualitative techniques frequently employed in social research. To our knowledge this is the first time these groups have been compared in this way.

\section{Methods}

PATIENTS

We studied 50 patients with severe COPD (forced expiratory volume in one second $\left(\mathrm{FEV}_{1}\right)<0.751$ and at least one admission for hypercapnic respiratory failure) and 50 patients with inoperable non-small cell lung cancer (NSCLC). None of the patients with NSCLC were receiving radiotherapy or chemotherapy at the time of interview and none were thought to have significant airflow obstruction. Patients with COPD were identi- fied from outpatient records of patients attending for follow up at Hull chest clinic. In the NSCLC group about one third were attending Hull chest clinic and the rest were identified from the database of the department of radiotherapy (that is, most patients had been referred for and received palliative radiotherapy). The lower age limit was 60 years. Written informed consent was obtained from all patients. The study was approved by the Hull and East Riding Research ethics committee. Patients were interviewed in their own homes by a single interviewer (JG). At the time of interview patients performed spirometric tests (Microspirometer MicroPlus, Micromedical Ltd, UK).

QUALITY OF LIFE INSTRUMENTS

In order to measure disease specific health status we used validated disease specific quality of life (QoL) tools. Patients with COPD were asked to complete the St George's Respiratory Questionnaire (SGRQ) ${ }^{11}$ and patients with NSCLC were given the EORTC Core QoL Questionnaire (QLQ-C30) and Lung Cancer Supplement (QLQ-LC13). ${ }^{12}$

Both groups were also assessed using generic health related QoL tools: the MOS Short Form-36 Health Survey (SF-36) ${ }^{13}$; the Nottingham Extended Activities and Daily Living (EADL) Scale ${ }^{14}$; and the Hospital Anxiety and Depression Scale (HADS). ${ }^{15}$ The SF-36 has been used in many cancer studies ${ }^{16}$ and has been validated in COPD. ${ }^{17}$ In addition, it has been proved to be robust for the comparison of patients with different conditions in a variety of settings. ${ }^{18}$ The EADL has been used in patients with severe COPD ${ }^{19}$ but not in cancer patients. The HADS does not rely on the presence of somatic symptoms which may characterise physical illness, and is therefore a valid global assessment of psychological morbidity in hospital patients. ${ }^{1520}$

\section{ASSESSMENT OF MEDICAL/SOCIAL CARE}

A semi-structured interview was designed to ascertain the views of patients regarding the quantity and quality of medical and social care received and the amount of information they had been given on their disease and treatment. Patients were asked to grade their satisfaction on a categorical scale of 1-7 where 7 represented "extremely satisfied" and 1 "extremely dissatisfied". General practice and hospital documentation were used to help verify the self-reported data from 20 patients chosen randomly from each group, particularly in relation to medication, aids and appliances, and social support.

ANALYSIS OF DATA

The interview data were coded and, with the QoL data, analysed using the Statistical Package for the Social Sciences (SPSS). All data were visually analysed. Parametric data were analysed by analysis of variance (ANOVA) and non-parametric data by the Wilcoxon signed rank test and Mann-Whitney U test. Qualitative data were scrutinised using social research techniques including categori- 
Table 1 Mean (SD) demographic characteristics of the patient populations

\begin{tabular}{lllll}
\hline & Age (years) & $M / F(\%)$ & $F E V_{1}(l)$ & KPI \\
\hline COPD & $70.5(5.5)$ & $44 / 56$ & $0.52(0.17)(\mathrm{n}=49)$ & $62.5(10.4)$ \\
NSCLC & $71.4(6.5)$ & $72 / 28$ & $1.47(0.39)(\mathrm{n}=42)$ & $66.9(9.2)$ \\
& $\mathrm{p}=0.436$ & & $\mathrm{p}<0.0001$ & $\mathrm{p}<0.05^{\star}$ \\
\hline
\end{tabular}

Forced expiratory volume in one second $\left(\mathrm{FEV}_{1}\right)$ and Karnofsky Performance Index (KPI) were used to help define the groups.

KPI scale 0-100: a score of 50-80 indicates severe disability with a lower score indicating worsening performance.

*Mann-Whitney U test.

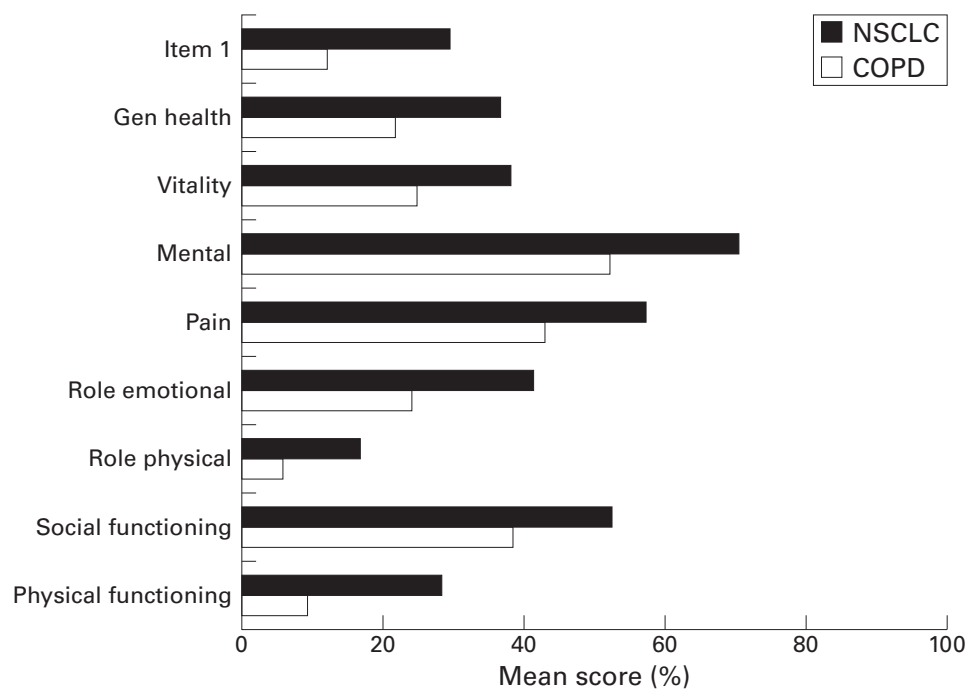

Figure 1 Mean SF-36 scores (scale 0-100\%). All dimensions except roles physical and emotional $=p \leqslant 0.05$ (Mann-Whitney U test). Gen health = general health;item 1 refers to how patients rate their general health. A higher score indicates a more favourable health status.

sation, theme development, and matrix building. ${ }^{21}$ These methods of qualitative analysis are designed to impose order on the mass of interview data by organising it into relevant categories which are directly related to the original research questions-for example, what the respondent says about "information provision". This is followed by a process of summarising, comparing, and then contrasting the data within and across the categories. Themes (patterns in the data) are then identified for each category and for the overall data set. Together with relevant quotations, the themes are placed into the relevant cells of a matrix and visually analysed. Inferences are then conceptualised and conclusions drawn.

\section{Results}

PATIENT CHARACTERISTICS

A total of 81 patients with COPD and 96 with NSCLC were selected from case note and hospital database review as suitable for recruitment. Of these, eight with COPD and 16 with NSCLC refused to take part, largely because of the severity of their illness, seven and 11, respectively, had died in the previous six months, and the rest did not respond, leaving 50 subjects in each group. Apart from a higher number of men who declined to take part in the study, the demographic characteristics did not appear to influence the recruitment of respondents.

The two groups of patients were similar in terms of age and performance status (table 1); the mean $\mathrm{FEV}_{1}$ for the COPD group was well
Table 2 Depression subscale of Health Anxiety and Depression Scale (HADS) for the two groups

\begin{tabular}{lll}
\hline & Mean $(S D)$ & Range \\
\hline COPD & $10.18(3.95)$ & $3-21$ \\
NSCLC & $7.22(5.16)$ & $0-20$ \\
& $\mathrm{p}<0.01$ & \\
\hline
\end{tabular}

Scale $0-21: \geqslant 8-10$ indicative of clinical depression. ${ }^{15}$

Table 3 Anxiety subscale of the Hospital Anxiety and Depression Scale for the two groups

\begin{tabular}{lll}
\hline & Mean $(S D)$ & Range \\
\hline COPD & $11.44(4.76)$ & $1-20$ \\
NSCLC & $7.20(5.25)$ & $0-21$ \\
& $\mathrm{p}<0.0001$ & \\
\hline
\end{tabular}

Scale $0-21: \geqslant 8-10$ indicative of clinical anxiety. ${ }^{15}$

below the entry criterion of 0.751 and the mean $\mathrm{FEV}_{1}$ for the NSCLC group was significantly higher, indicating that severe COPD was not present in the patients with NSCLC. The median (range) interval between diagnosis and interview for the COPD group was 6 (1-30) years and for the NSCLC group 1 (0.1-8) year; $50 \%$ of the patients with COPD and $64 \%$ of those with NSCLC had full time carers (usually the spouse) living with them.

QUALITY OF LIFE DATA

Disease specific questionnaires

The SGRQ has four domains: symptoms, impacts, activities, and a total score. It rates health status as a percentage, a higher score indicating a worse QoL. The mean (SD) SGRQ scores for the COPD group were: symptoms 80 (14)\%; activities 85 (13)\%; impacts $62(20) \%$; total 72 (14)\%. The EORTC has three main "functioning" categories (physical, social, and emotional) and a global score. A higher score (as a percentage) indicates a better QoL. The mean (SD) scores for patients with NSCLC were: physical 42 (23)\%; social 53 (35)\%; emotional 70 (30)\%; global $45(27) \%$.

\section{Generic questionnaires}

The EADL has a scale of 0-22, a lower score representing greater limitation. The COPD group had a significantly worse mean (SD) EADL score than the NSCLC group (9.4 (4.1) vs $11.3(4.2), p<0.05)$. For all the dimensions of the SF-36 questionnaire apart from roles emotional and physical, patients with COPD had significantly worse (lower) mean scores than those with NSCLC (fig 1). Similarly, the HADS scores were significantly worse (higher) in the COPD group than in the NSCLC group, with the mean scores reaching a level suggesting clinically relevant disease in the former but not the latter group (tables 2 and 3 ).

In addition, the interview data revealed that $82 \%$ of patients with COPD were housebound compared with $36 \%$ of those with NSCLC, and $36 \%$ and $10 \%$, respectively, were largely chairbound (only able to move from one room to another with great difficulty). Furthermore, while most of the patients with NSCLC felt able to accept their illness and retain a fairly positive outlook, most of the patients with COPD were "frustrated", "angry", and expressed anxiety about attacks of dyspnoea. 
Table 4 Mean (SD) number of outpatient follow up visits, emergency admissions, and GP visits per year

\begin{tabular}{llll}
\hline & $\begin{array}{l}\text { Outpatient follow } \\
\text { up visits }\end{array}$ & $\begin{array}{l}\text { Emergency } \\
\text { admissions }\end{array}$ & GP visits \\
\hline COPD & $3.2(1.3)$ & $4.1(4.0)$ & $8.5(6.8)$ \\
NSCLC & $5.2(10.5)$ & $1.3(1.2)$ & $10.7(11.2)$ \\
& $\mathrm{p}=0.2$ & $\mathrm{p}<0.001$ & $\mathrm{p}=0.01$ \\
\hline
\end{tabular}

MEDICAL CARE

The mean numbers of outpatient attendances were similar in both groups (table 4 ); $80 \%$ of patients with NSCLC had received palliative radiotherapy in the previous 12 months, accounting for most of their outpatient visits. Almost all of the outpatient attendances in the COPD group were follow up visits to the chest clinic. However, the numbers of emergency admissions and GP visits per year differed between the groups, with the COPD patients having significantly more emergency admissions per year (largely due to exacerbations) and the NSCLC patients seeing their GP more often (usually because of newly emerging symptoms). The most commonly used medications in the COPD group were bronchodilators (94\%), long term oxygen therapy (LTOT) $(40 \%)$, and inhaled or oral steroids (38\%). In the NSCLC group opiate analgesia (36\%) was the most commonly used medication. Although $90 \%$ of patients with COPD and $52 \%$ of those with NSCLC had scores suggesting depression or anxiety, only $4 \%$ in each group received further assessment and treatment.

The majority of patients in both groups (86\% COPD, 84\% NSCLC) were generally satisfied with the quantity of medical care and attention received. The level of satisfaction with medical care (measured by a seven point rating scale) did not differ between the groups, with mean (SD) scores of 5.9 (1.4) and 5.7 (1.4) in the COPD and NSCLC groups, respectively. These scores approximated to the response "very satisfied". However, in the interviews most patients in both groups qualified their satisfaction rating by stating that they felt they were receiving all the treatment available, but that such treatment was limited in what it could offer to meet their physical and wider needs.

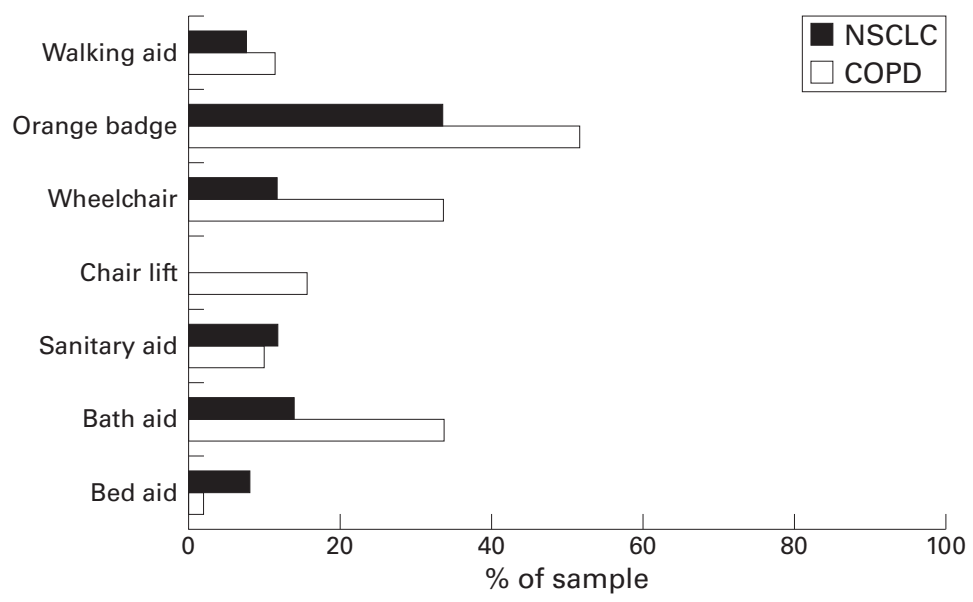

Figure 2 Proportion of each group receiving various aids and appliances.
SOCIAL AND COMMUNITY CARE

The numbers receiving financial help due to their illness were similar (76\% of the COPD group and $68 \%$ of NSCLC patients). A higher proportion of patients with COPD (40\%) than with NSCLC (10\%) felt that the financial support received came late in their illness and was delayed from when they had first become eligible (often 2-4 years after diagnosis). Similar proportions of respondents in both groups (74\% COPD, 66\% NSCLC) had aids and appliances to help cope with their illness (fig 2).

Although for most aids and appliances patients with COPD received more than those with NSCLC, just over half of the COPD respondents received an Orange Badge (for disabled parking), $16 \%$ had a chairlift ( $6 \%$ had these installed by social services, the others were self-purchased), 34\% received bath aids, and only one patient had bed aids. These figures should be set against the fact that most of the patients with COPD were housebound and nearly a third were chairbound. Furthermore, more of the patients with COPD (50\%) than with lung cancer $(32 \%)$ felt that they could benefit from a better provision of aids and appliances. Finally, $72 \%$ of patients with COPD and $52 \%$ of those with NSCLC reported a lack of information regarding the possible social benefits and services they could receive. This often resulted in delay in receiving the support to which the patient was entitled.

Use of community agencies differed mainly in terms of specialist palliative care services (fig 3). Nearly one third of patients with NSCLC (30\%) received help from a Marie Curie nurse, Macmillan nurse, or hospice centre and a further $56 \%$ had been offered or were aware of the availability of these services. In contrast, none of the patients with COPD received or were offered access to these agencies or any equivalent service. Almost a quarter of patients with COPD (24\%) were visited on an ad hoc basis by a community based respiratory support nurse (RSN) whose primary responsibility was tuberculosis contact tracing. Most of these patients found this to be their main source of emotional support, nursing care, and help in applying for social benefits. Although some patients with COPD received a visit from a district nurse, this was often as a result of nursing needs unrelated to their illness-for example, to re-dress a wound-or may only have occurred immediately following discharge from hospital. Sixty percent of patients with COPD and $16 \%$ of those with NSCLC were seen by a hospital social worker before discharge from hospital in order to assess whether the patient required apparatus for the home. However, only a small minority in each group ever received further assessment from a community social worker. Patients with COPD were more likely than those with NSCLC to be dissatisfied with the extent of help from social services and other social agents (36\% vs $12 \%$ ).

INFORMATION PROVISION

A major area of dissatisfaction for patients in both groups was the perceived inadequate provision of information regarding their illness, its 


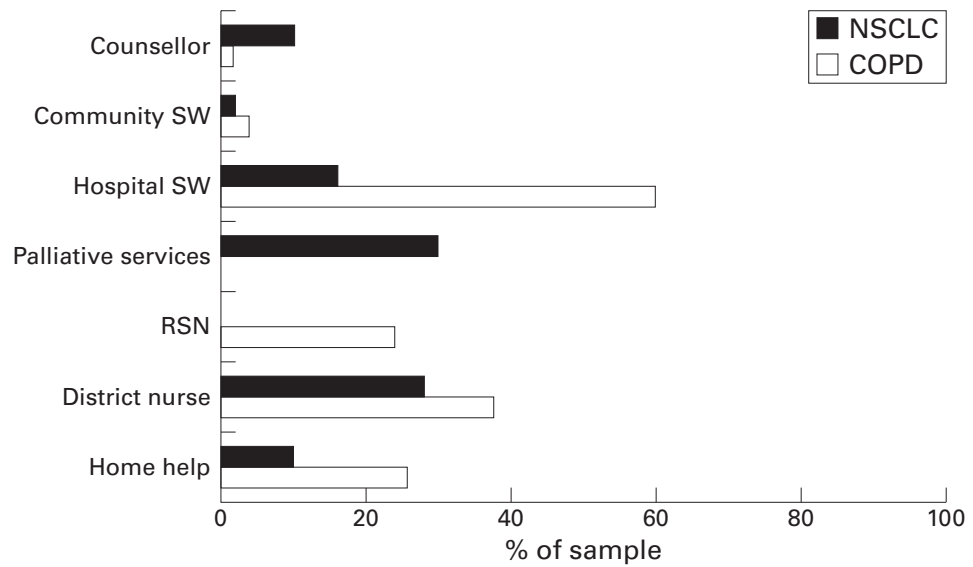

Figure 3 Proportion of each group receiving various community care services. $R S N=$ respiratory support nurse; $S W=$ social worker.

management, and the type of social help available. Thirty percent of COPD patients and $34 \%$ of NSCLC patients felt that diagnostic information had been lacking or given insensitively and $78 \%$ and $80 \%$, respectively, said that they did not receive enough information regarding their prognosis or future management from their hospital doctors. In many cases the patients' awareness of their prognosis was brought about through their own experiences-for example, emergency admissions - and through conversations with other health personnel such as district nurses. Some patients with COPD reported that, as they had received little information, they felt unprepared for their current poor state of health. Other patients with COPD complained that they had not understood the terms used by their doctors during initial consultations-for example, "emphysema" or "COPD"-nor their implications. In the NSCLC group the main area of concern was the absence of information regarding treatment. For example, some patients spoke of being "left in a vacuum" while doctors decided whether palliative radiotherapy was appropriate. A minority gave accounts of doctors contradicting one another with regard to illness management. Furthermore, some cancer patients felt that the initial diagnosis had been given insensitively or abruptly.

However, patients' information needs were diverse and sometimes ambiguous and contradictory. Although $42 \%$ of patients with COPD and $40 \%$ with NSCLC suggested they wanted more information regarding their illness and future management at the time of interview, very few wanted detailed information and implied that an accurate description of their likely progression would be distressing (a typical comment being "what you don't know can't hurt you"). In the NSCLC group 24\% were frustrated that the doctor had avoided the word "cancer" while $18 \%$ felt more comfortable with less fearful words such as "shadow" or "growth". Many patients appeared to be in denial; while almost all of the patients in both groups knew that they had a life threatening illness, $42 \%$ with NSCLC and $26 \%$ with COPD said or implied that they did not know whether they would die from their disease. When asked why they did not request more information from their doctors, most patients stated that they felt it was inappropriate and that such discussions should be initiated by their specialists.

\section{Discussion}

Current best practice ${ }^{22}$ demands that patients with malignancy have access not only to oncological expertise to maximise survival prospects but also to a multidisciplinary palliative approach which focuses on how best to support patients and their families through the illness while maintaining reasonable quality of life. Key aspects include symptom control, recognition and management of psychological problems, good communication skills, and helping patients and carers with financial and practical issues. We postulated that patients with severe COPD might benefit from this approach and set out to compare their needs with those of a group of patients who were the traditional recipients of this type of holistic care. We confirmed that these severely ill patients with COPD had a very poor QoL in terms of emotional, social, and physical functioning and that there was a high prevalence of unrecognised psychological disorder and unmet information needs.

The groups were grossly similar in terms of their poor performance status as measured by the Karnofsky scale, although there was a small difference in favour of the NSCLC patients. The participants were drawn from different consultants' case loads and uniformly satisfied the selection criteria. Further, their disease specific QoL scores were comparable with those of previously studied patients with the same diseases. ${ }^{23}{ }^{24}$ Although we recognise that the patient populations might appear somewhat atypical, demographic characteristics did not affect the selection process nor the numbers who agreed to be interviewed. The sex distribution in the COPD group reflects the high local prevalence of this disease in women and East Riding Health Authority figures confirm the near equality of admission by sex for COPD (ICD-10 codes J40-44). The patients with NSCLC appeared to have a relatively lengthy survival from diagnosis. Although not explicitly excluded, we were reluctant to approach people who were rapidly deteriorating or were in the process of receiving treatment. In this group some patients had initially undergone what was thought to be curative surgery (and then relapsed) while many of the remainder were identified from department of radiotherapy records and were fit enough to have received and recovered from palliative treatment. Because of these factors many of the patients were in a relatively stable state at the time of interview. It is our contention that the patient population was a representative sample of patients with end stage COPD and longer surviving, but inoperable, NSCLC. Although the data are based on interviews with patients, GP and hospital documentation were used to help verify data acquired from a random sample of patients. Our scrutiny of medical records 
(representing 20 patients in each group) essentially confirmed the accuracy of the selfreported received medical and social care.

The patients with COPD had significantly worse health status (physical, social, and emotional) and activities of daily living than those with NSCLC. Further, they had symptoms of anxiety and depression to a significantly greater extent than the patients with NSCLC. There was, however, variability among patients with regard to their symptoms and limitations, explaining the rather high standard deviations found in both groups for the SF-36 and HADS. Although widely used, the HADS is a screening tool rather than a diagnostic instrument and lacks the specificity of other depression or anxiety scales such as the Present State Examination. Despite the fact that previous studies have suggested an unexpectedly high incidence of depression in patients with COPD, a recent systematic review was more cautious in its conclusions. ${ }^{25}$

Considering the poor prognosis of lung cancer, it is perhaps surprising that the incidence of psychological disturbance was much lower in the NSCLC group. It is possible that the NSCLC patients who consented to interview were less psychologically affected by their illness than those who refused to take part in the study, but we did not have the opportunity to characterise the non-participants in order to confirm or refute this, and the explanation could be applied equally to the COPD group. It might also be argued that the lung cancer patients were interviewed at a time when the disease had not yet had its full impact and it could be anticipated that a group of medium term survivors might be less psychologically affected than those with rapidly progressive disease. However, these patients were relatively advanced in terms of the natural history of their tumours and a third were requiring opiate analgesia. The interview data indicated that the illness had not restricted the daily lives of the patients with NSCLC as much as those with COPD, perhaps enabling the former to adopt a more positive outlook.

Both groups of patients seemed generally satisfied with the medical treatment they had received. However, many patients recognised the limits of medical care and it appeared inadequate in terms of meeting the psychological requirements and overall holistic needs of both sets of patients. Of considerable concern is that, despite the high level of possible need subsequently identified by the screening tool, very few subjects had received any assessment or treatment for anxiety and depression (as indicated by patient recall, drug prescriptions, and entries in the medical notes). The reluctance of doctors to diagnose and treat mental health problems in those with physical illness is widely recognised in other contexts ${ }^{26}{ }^{27}$ and may be partly due to the view that "reactive" psychological morbidity is an inevitable consequence of chronic physical disease and is refractory to treatment. Unfortunately, many of the biological symptoms of depression such as fatigue, weight loss, and sleep distur- bance are common in advanced COPD and may well have been ascribed to the latter.

Information provision was inadequate in terms of both content and delivery. The deficiencies experienced by the patients in this study may be partly explained by the variation and complexity in patients' information needs and, particularly in the NSCLC group, the likelihood that some might have been in denial. Patients described the seeking of reassurance and their disappointment when their doctors were unable to give it. Some wished they had received more information, irrespective of the distressing content, while others did not wish to know. In the former case it may be that the patients' need for more detailed information conflicted with the doctors' need to protect them and to retain an optimistic outlook. ${ }^{28}$ The complexity of patients' information requirements and the demonstrated failure to meet them confirms the primacy of communication skills in everyday practice and the necessity of their refinement at all levels of seniority.

The paramount difference in care between the groups was in social and community support. Compared with the lung cancer group, the patients with COPD received more aids and appliances but, because of their greater physical limitation, this did not necessarily meet their needs and they were more likely to be dissatisfied with the care received. The most obvious disparity was that the patients with NSCLC had ready access to specialist palliative care services whereas those with COPD did not have an equivalent system of support. The patients with COPD experienced a lack of regular needs assessment at home and their palliative care requirements remained unrecognised. In some cases, however, the respiratory support nurse, working outside her usual remit, provided valuable practical help and advice. Two sets of guidelines have briefly considered the process of providing some form of pastoral support to patients with severe COPD in the community. ${ }^{49}$ Both advocate a respiratory health worker as the means to deliver advice on "psychosocial and respiratory problems and to improve compliance ...". The optimum use of such personnel has yet to be defined and there is surprisingly little known about their usefulness. One controlled study of the impact of a respiratory health worker showed increased levels of patient knowledge and satisfaction and a trend to reduced mortality, but longer hospital stays and no reduction in admission rate. ${ }^{30}$ Current estimates suggest that there are fewer than 300 specialist respiratory nurses in the UK (C Fehrenbach, personal communication) and local priorities may be focused on other diseases such as asthma or tuberculosis. Given the prevalence of severe COPD in the community, these numbers are clearly inadequate.

In recent years pulmonary rehabilitation has been more widely introduced, usually in a secondary care setting. These programmes are variably constituted but are commonly multidisciplinary and usually directed at those with at least moderately severe COPD. In addition to a physical training component, some form of 
psychological support is incorporated and many programmes have educational, social work, and occupational therapy inputs. Most studies suggest improvements in exercise tolerance, quality of life, and coping skills after completing treatment, ${ }^{31}$ but the benefits may not be sustained and the numbers of patients able to receive treatment at any one time may be limited for practical reasons. Both interventions appear to address some of the problems faced by patients with COPD, although the number of patients with the potential to benefit far exceeds capacity.

Are these results generalisable to the rest of the UK? There appear to be no available data on pastoral care for patients with COPD, but intuitively the picture is unlikely to be uniform and will depend on the local prevalence of other respiratory diseases, existing resources and, to a degree, the perceived priorities of secondary care and the health authority. The care of cancer patients is likely to depend on similar factors and also the proximity to a hospice or cancer centre and the nature of the community served. From our observations there is no reason to believe that our service is very different from that available elsewhere but, in order to test this, it would be necessary to repeat a similar study in another centre.

The COPD population we studied received a system of support which was fragmentary and largely dependent on initiatives from secondary care. While social care provision for the patients with NSCLC was suboptimal-for example, lack of information regarding social benefits and illness management-these patients had the safety net of a more holistic approach provided by a team with palliative care skills. The absence of a similar model for patients with COPD is partly historical, but the hospice movement is now beginning to recognise that the disability, progressive nature, and poor prognosis of such chronically ill patients makes them suitable candidates for this type of holistic care. Nevertheless, COPD does not appear to be regarded as a high profile illness by the public or health professionals. It is tempting to speculate that the common view of this condition as a selfinflicted disease and its lengthy time course have allowed it to be relegated in the hierarchy of areas for action and concern.

In conclusion, this study confirms our hypothesis that patients with end stage COPD have palliative care needs which remain unaddressed. We would argue that many of them have unmet information requirements, unrecognised psychological problems, a very poor overall QoL, and a need for palliative care which parallels that of patients with lung cancer. This study is a further contribution to the growing body of research which suggests that patients with end stage COPD require more holistic and patient centred care than they are currently receiving.

We thank the following for their invaluable advice: Professor $\mathrm{P}$ Jones, Professor P Calverley, Dr M McCarthy, Dr N Small, Mr R Francis, Mr E Gardner, Mrs P Webb, Dr J Addington-Hall, R Francis, Mr E Gardner, Mrs P Webb, Dr J Addington-Hall,
Mrs R Lall. We also thank Dr A Arnold, Dr D McGivern, Mr M Mrs R Lall. We also thank Dr A Arnold, Dr D McGivern, Mr M
Cowen, Dr M Holmes and Dr R Dealey for allowing us access to their patients. Finally, we wish to extend our appreciation to to their patients. Finally, we wish
the patients who kindly took part. This study was funded by a Northern and Yorkshire Regional
R\&D Directorate grant.

1 Williams SJ. Chronic respiratory illness. London: Routledge, 1993.

2 Cox BD. Blood pressure and respiratory function. In: The health and lifestyle survey. Preliminary report of a nationwide survey of the physical and mental health attitudes and lifestyle of a random sample of 9003 British adults. London: Health Promotion Research Trust, 1987: 17-33.

3 Strachan DP. Epidemiology: a British perspective. In: Calverley P, Pride N, eds. Chronic obstructive pulmonary disease. London: Chapman and Hall, 1995: 47-67.

4 British Thoracic Society.BTS guidelines for the management of chronic obstructive pulmonary disease. Thorax 1997;52(Suppl 5):S5.

5 McSweeny AJ, Grant I, Heaton RK, et al. Life quality of patients with chronic obstructive pulmonary disease. Arch Intern Med 1982;142:473-8.

6 Medical Research Council Working Party. Long term domiciliary oxygen therapy in chronic hypoxic cor pulmonale complicating chronic bronchitis and emphysema. Lancet 1981;1:681-6.

7 Royal College of Physicians (Research Unit). Palliative care: guidelines for good practice and audit measures. $\mathcal{F} \mathrm{R}$ Coll Physicians Lond 1991;25:325-8.

8 Eve A, Smith AM, Tebbit P. Hospice and palliative care in the UK 1994-5, including a summary of trends 1990-5. Palliative Med 1997;11:31-43.

9 McCarthy M, Lay M, Addington-Hall J. Dying from heart disease. F $R$ Coll Physicians Lond 1996;30:325-8.

10 Shee CD. Palliation in chronic respiratory disease. Palliative Med 1995;9:3-12.

11 Jones PW, Quirk FH, Baveystock CM. A self complete measure of health status for chronic airflow limitation. Am Rev Respir Dis 1992;145:1321-7.

12 Bergman B, Aaronson NK, Ahmedzai S, et al for the European Organisation for Research and Treatment of Cancer (EORTC) Study Group on Quality of Life. The EORTC QLQ LC13: a modular supplement to the EORTC Core Quality of Life Questionnaire (QLQ-C30) for use in lung cancer clinical trials. Eur f Cancer 1994;30A:635-42.

13 Ware JE, Sherbourne CD. The MOS 36-item short-form Ware JE, Sherbourne CD. The MOS 36 -item short-form
health survey (SF-36): 1. Conceptual framework and item health survey (SF-36): 1. Conceptual
selection. Med Care 1992;30:473-83.

14 Yohannes AM, Roomi J, Connolly MJ. Elderly people at home disabled by chronic obstructive pulmonary disease. Age Ageing 1998;27:523-5.

15 Zigmond AS, Snaith RP. Hospital anxiety and depression scale. Acta Psychiatry Scand 1983;67:361-70.

16 Kurtz ME, Kurtz JC, Stommel M, et al. Loss of physical functioning among geriatric cancer patents: relationships to cancer site, treatment, comorbidity and age. Eur $\mathcal{F}$ Cancer 1997;33:2352-8

17 Viramontes JL, O'Brien B. Relationship between symptoms and health-related quality of life in chronic lung disease. $\mathcal{F}$ Gen Intern Med 1994;9:46-8.

18 Brazier JE, Harper R, Jones NMB, et al. Validating the SF-36 health survey questionnaire: new outcome measure for primary care. $B M \mathcal{F}$ 1992;305:160-4.

19 Okubidejo AA, O'Shea L, Jones PW, et al. Home assessment of activity of daily living in patients with severe chronic obstructive pulmonary disease on long-term oxygen obstructive pulmonary disease on

20 Clark A, Fallowfield LJ. Quality of life measurement in patients with malignant disease: a review. $\mathcal{f} R$ Soc Med 1986;79:165-9

21 Miles MB, Huberman AM. Qualitative data analysis: an expanded sourcebook. London: Sage, 1994.

22 Effective Health Care. Management of lung cancer.Volume 4, 3. University of York: NHS Centre for Reviews and Dissemination, 1998;1-12.

23 Meecham Jones DJ, Paul EA, Jones PW, et al. Nasal pressure support ventilation plus oxygen compared with oxygen therapy alone in hypercapnic COPD. Am 7 Respir Crit Care Med 1995;152:538-44.

24 Aaronson NK, Ahmedzai S, Bergman B, et al. The EORTC QLQ-C30: a quality instrument for use in international clinical trials in oncology. F Natl Cancer Inst 1993;85:36575 .

25 van Ede L, Yzermans CJ, Brouwer HJ. Prevalence of depression in patients with chronic obstructive pulmonary depression in patients with chronic obstructive pulmo

26 Hardman A, Maguire P, Crowther D. The recognition of psychiatric morbidity on a medical oncology ward. $f$ Psychosomatic Res 1989;33:235-9.

27 Bridges KW, Goldberg DP. Psychiatric illness in inpatients with neurological disorders: patients' views on discussion of emotional problems with neurologists. BMF 1985;291: 656-8.

28 Faulkner A. Communication with patients, families, other professionals: ABC of palliative care. BMF 1998;316:1302.

29 Royal College of Physicians. Disabling Chest Disease: Prevention and Care. F R Coll Physicians Lond 1981;15:69-

30 Cockroft A, Bagnall P, Heslop A, et al. Controlled trial of respiratory health worker visiting patients with chronic respiratory disability. BMF 1987:294:225-8.

31 American College of Chest Physicians/American Association of Cardiovascular and Pulmonary Rehabilitation ation of Cardiovascular and Pulmonary Rehabilitation AACVPR evidence-based guidelines. Chest 1997;112: 1363-96. 